\title{
A study of existence and multiplicity of positive solutions for nonlinear fractional differential equations with nonlocal boundary conditions
}

\author{
Noureddine Bouteraa and Slimane Benaicha
}

\begin{abstract}
This paper deals with the existence, uniqueness and the multiplicity of solutions for a class of fractional differential equations boundary value problems involving three-point nonlocal Riemann-Liouville fractional derivative and integral boundary conditions. Our results are based on some well-known tools of fixed point theory such as Banach contraction principle, fixed point index theory and the Leggett-Williams fixed point theorem. As applications, some examples are presented at the end to illustrate the main results.
\end{abstract}

Mathematics Subject Classification (2010): 34A08, 26A33, 34A60.

Keywords: Positive solution, fractional differential equations, existence, multiplicity, nonlocal boundary, Green's function.

\section{Introduction}

In this paper, we are interested in the existence of solutions for the nonlinear fractional differential equation

$$
D_{0^{+}}^{\alpha} u(t)+a(t) f(t, u(t))=0, \quad t \in(0,1),
$$

subject to the boundary condition

$$
u^{(i)}(0)=0, i \in\{0,1,2\}, D_{0^{+}}^{\beta} u(1)=\lambda I_{0^{+}}^{\beta} u(\eta),
$$

where $D_{0^{+}}^{\alpha}, D_{0^{+}}^{\beta}$ are the standard Riemann-Liouville fractional derivative of order $\alpha \in(3,4], \beta \in[2,3], I_{0+}^{\beta}$ is the stantard Riemann-Liouville fractional integral of order $\beta \in[2,3]$. 
Due to the fact that the tools of fractional calculus has numerous applications in various disciplines of science and engineering such as physics, mechanics, chemistry, biology, aerodynamics, electrodynamics of complex medium, polymer rheology, Bode's analysis of feedback amplifiers, capacitor theory, electrical circuits, electroanalytical chemistry, control theory, fitting of experimental data, involves derivatives of fractional order. In consequence, the subject of fractional differential equations is gaining much importance and attention. Therefore, there have been many papers and books dealing with the theoretical development of fractional calculus and the solutions or positive solutions of boundary value problems for nonlinear fractional differential equations. For more details we refer the reader to $[10,19,21]$ and the references cited therein.

Many mathematicians show strong interest in fractional differential equations and many wonderful results have been obtained. The techniques of nonlinear analysis, as the main method to deal with the problems of nonlinear fractional differential equations, plays an essential role in the research of this field, such as establishing the existence and the uniqueness or the multiplicity of solutions to nonlinear fractional differential equations boundary value problems, see $[2,5,7,9,11,14,16,18]$ and the references therein.

In [17], the authors studied the existence of positive solutions to the following fractional boundary value problem

$$
\left\{\begin{array}{l}
D_{0^{+}}^{\alpha} u(t)+h(t) f(t, u(t))=0, \\
u(0)=u^{\prime}(0)=u^{\prime \prime}(0)=0, \quad u(1)=\lambda \int_{0}^{\eta} u(\eta) d s
\end{array}\right.
$$

where $D_{0^{+}}^{\alpha}$ are the standard Riemann-Liouville fractional derivative of order $\alpha \in$ $(3,4], \eta \in(0,1]$, and $0 \leq \frac{\lambda \eta^{\alpha}}{\alpha}<1$.

In [22], the authors studied the boundary value problems of the fractional order differential equation:

$$
\left\{\begin{array}{l}
D_{0^{+}}^{\alpha} u(t)=f(t, u(t))=0, \quad t \in(0,1), \\
u(0)=0, \quad D_{0^{+}}^{\beta} u(1)=a D_{0^{+}}^{\beta} u(\eta),
\end{array}\right.
$$

where $1<\alpha \leq 2,0<\eta<1,0<a, 0<\beta \leq 1, f \in C([0,1] \times[0, \infty),[0, \infty))$ and $D_{0^{+}}^{\alpha}, D_{0^{+}}^{\beta}$ are the standard Riemann-Liouville fractional derivative of order $\alpha, \beta$. They obtained the multiple positive solutions by the Leray-Schauder nonlinear alternative and the fixed point theorem on cones.

In 2017, Benaicha and Bouteraa [3] studied the existence and uniqueness of solutions for nonlinear fractional differential equation

$$
{ }^{c} D^{\alpha} u(t)=f\left(t, u(t), u^{\prime}(t)\right), \quad t \in J=[0,1]
$$

subject to three-point boundary conditions

$$
\left\{\begin{array}{l}
\beta u(0)+\gamma u(1)=u(\eta) \\
u(0)=\int_{0}^{\eta} u(s) d s \\
\beta^{c} D^{p} u(0)+\gamma^{c} D^{p} u(1)={ }^{c} D^{p} u(\eta)
\end{array}\right.
$$


where $2<\alpha \leq 3,1<p \leq 20<\eta<1, \beta, \gamma \in \mathbb{R}^{+}, f:[0,1] \times \mathbb{R} \times \mathbb{R} \rightarrow \mathbb{R}$ is a continuous function and ${ }^{c} D^{\alpha}$ denotes the Caputo fractional derivative of order $\alpha$.

In 2018, Bouteraa and Benaicha [6] interested in the existence of solutions for the nonlinear fractional differential equation

$$
D_{0^{+}}^{\alpha} u(t)+f(t, u(t))=0, \quad t \in(0,1),
$$

subject to the boundary conditions

$$
u^{(i)}(0)=0, i \in\{0,1, \ldots, n-2\}, D_{0^{+}}^{\beta} u(1)=\sum_{j=1}^{p} a_{j} D_{0^{+}}^{\beta} u\left(\eta_{j}\right),
$$

where $D_{0^{+}}^{\alpha}, D_{0^{+}}^{\beta}$ are the standard Riemann-Liouville fractional derivative of order $\alpha(n-1, n], \beta \in[1, n-2]$ for $n \in \mathbb{N}^{*}$ and $n \geq 3$ and $f \in C((0,1) \times \mathbb{R}, \mathbb{R})$ is allowed to be singular at $t=0$ and/or $t=1$ and $a_{j} \in \mathbb{R}^{+}, j=1,2, \ldots, p, 0<\eta_{1}<\eta_{2}<\ldots<$ $\eta_{p}<1$, for $p \in \mathbb{N}^{+}$. The existence and uniqueness of positive solutions for the above nonlocal boundary value problem obtained by applying the iterative method.

Inspired and motivated by the works mentioned above, we focus on the existence of positive solutions for the nonlocal boundary value problem (1.1) - (1.2). The paper is organized as follows. In Section 2, we recall some preliminary facts that will be need in the sequel. In Section 3, we establish the existence, uniqueness and multiplicity of the positive solutions for boundary value problem (1.1) - (1.2) by applying some wellknown tools of fixed point theory such as Banach contraction principle, fixed point index theory and the Leggett-Williams fixed point theorem and we give two examples to illustrate our results.

\section{Preliminaries}

In this section, we recall some definitions and facts which will be used in the later analysis.

Definition 2.1. ([20]) Let $E$ be a real Banach space. A nonempty closed set $K \subset E$ is said to be a cone provided that

(i) $c_{1} u+c_{2} v \in K$ for all $c_{1} \geq 0, c_{2} \geq 0$, and

(ii) $u \in K,-u \in K$ implies $u=0$.

Every cone $K$ induces an ordering in $E$ given by $u \leq v$ if and only if $v-u \in K$.

Definition 2.2. ([10,15]) The Riemann-Liouville fractional integral of order $\alpha>0$ of a function $u:(0, \infty) \rightarrow \mathbb{R}$ is given by

$$
I_{0^{+}}^{\alpha} u(t)=\frac{1}{\Gamma(\alpha)} \int_{0}^{t}(t-s)^{\alpha-1} u(s) d s, \quad t>0,
$$

where $\Gamma(\cdot)$ is the Euler gamma function, provided that the right side is pointwise defined on $(0, \infty)$. 
Definition 2.3. ([10,15]) The Riemann-Liouville fractional derivative order $\alpha>0$ of a continuous function $u$ is defined by

$$
D_{0^{+}}^{\alpha} u(t)=\frac{1}{\Gamma(n-\alpha)} \frac{d^{n}}{d t^{n}} \int_{0}^{t}(t-s)^{n-\alpha-1} u(s) d s, \quad t>0,
$$

where $\Gamma(\cdot)$ is the Euler gamma function and $n=\lceil\alpha\rceil+1,\lceil\alpha\rceil$ denotes the integer part of number $\alpha$, provided that the right side is pointwise defined on $(0, \infty)$.

Lemma 2.4. ([10]) (i) If $u \in L^{p}(0,1), 1 \leq p \leq+\infty, \beta>\alpha>0$, then

$$
D_{0^{+}}^{\alpha} I_{0^{+}}^{\beta} u(t)=I_{0^{+}}^{\beta-\alpha} u(t), D_{0^{+}}^{\alpha} I_{0^{+}}^{\alpha} u(t)=u(t), I_{0^{+}}^{\alpha} I_{0^{+}}^{\beta} u(t)=I_{0^{+}}^{\alpha+\beta} u(t) .
$$

(ii) If $\beta>\alpha>0$, then $D^{\alpha} t^{\beta-1}=\frac{\Gamma(\beta) t^{\beta-\alpha-1}}{\Gamma(\beta-\alpha)}$.

(iii) If $\alpha>0$ and $\gamma \in(-1,+\infty)$, then $I_{0^{+}}^{\alpha} t^{\gamma}=\frac{\Gamma(\gamma+1)}{\Gamma(\alpha+\gamma+1)} t^{\alpha+\gamma}$.

Lemma 2.5. ([10]) Let $\alpha>0$ and for any $y(\cdot) \in L^{1}(0,1)$. Then, the general solution of the fractional differential equation $D_{0^{+}}^{\alpha} u(t)+y(t)=0,0<t<1$ is given by

$$
u(t)=-\frac{1}{\Gamma(\alpha)} \int_{0}^{t}(t-s)^{\alpha-1} y(s) d s+c_{1} t^{\alpha-1}+c_{2} t^{\alpha-2}+\cdots+c_{n} t^{\alpha-n},
$$

where $c_{0}, c_{1}, \ldots, c_{n-1}$ are real constants and $n=\lceil\alpha\rceil+1$.

Now, let $0<d<l<r$ be given and let $\beta$ be a nonnegative continuous concave functional on the cone $K$ i.e.,

$$
\beta(\lambda u+(1-\lambda) v) \geq \lambda \beta(u)+(1-\lambda) \beta(v),
$$

for all $u, v \in K$ and $\lambda \in[0,1]$.

Define the convex sets $K_{l}$ and $K(\beta, l, r)$ by

$$
K_{l}=\{u \in K:\|u\|<l\},
$$

and

$$
K(\beta, l, r)=\{u \in K: l \leq \beta(u),\|u\| \leq r\} .
$$

The key tools in our approaches are the following fixed point theorem and lemmas

Theorem 2.6. (Leggett-Wiliams fixed point $($ See [20])) Let $E$ be a Banach space and $K \subset E$ be a cone in $E . T: \bar{K}_{c} \rightarrow \bar{K}_{c}$ be a completely continuous and $\beta$ be a nonnegative continuous concave functional on $K$ with $\beta(u) \leq\|u\|$ for all $u \in K_{c}$. Suppose there exist $0<d<l<r \leq c$ such that

(i) $u \in\{K(\beta, l, r): \beta(u)>l\} \neq \emptyset$ and $\beta(T u)>l$ for $u \in K(\beta, l, r)$,

(ii) $\|T u\|<d$ for $\|u\| \leq d$,

(iii) $\beta(T u)>l$ for $u \in K(\beta, l, c)$ with $\|T u\|>r$.

Then $T$ has at least three positive solutions $u_{1}, u_{2}, u_{3}$ satisfying

$$
\left\|u_{1}\right\|<d, \quad l<\beta\left(u_{2}\right), \quad\left\|u_{3}\right\|>d \text { and } \beta\left(u_{3}\right)<l .
$$

Lemma 2.7. (Krein-Rutman [20]) Let $K$ be a reproducing cone in a real Banach space $E$, and $L: E \rightarrow E$ be a compact linear operator with $L(K) \subseteq K$ and spectral radius $r(L)$. If $r(L)>0$, then there exists $\varphi \in K \backslash\{0\}$ such that $L \varphi=r(L) \varphi$. 
Lemma 2.8. (Fixed point index theory [20]) Let $E$ be a Banach space and $K$ is a cone in $E$ and $\Omega(K)$ is a bounded open subset in $K$. Furthermore, assume that $T: \overline{\Omega(K)} \rightarrow K$ is a completely continuous operator Then the following conclusion hold:

(i) there exists $u_{0} \in K \backslash\{0\}$ such that $T u+\lambda u_{0} \neq u$ for all $u \in \partial \Omega(K)$ and $\lambda \geq 0$, then the fixed point index $i(T, \Omega(K), K)=0$,

(ii) if $0 \in \Omega(K)$ and $T u \neq \lambda u$ for all $u \in \partial \Omega(K)$ and $\lambda \geq 1$, then the fixed point index $i(T, \Omega(K), K)=1$.

Lemma 2.9. Let $y(\cdot) \in C[0,1]$. Then the solution of the fractional boundary value problem

is given by

$$
\left\{\begin{array}{l}
D_{0^{+}}^{\alpha} u(t)+y(t)=0 \\
u^{(i)}(0)=0, i \in\{0,1,2\} \\
D_{0^{+}}^{\beta} u(1)=\lambda I_{0^{+}}^{\beta} u(\eta),
\end{array}\right.
$$

where

$$
u(t)=\int_{0}^{1} G(t, s) y(s) d s
$$

$$
G(t, s)= \begin{cases}\frac{-P \Gamma(\alpha-\beta) \Gamma(\alpha+\beta)(t-s)^{\alpha-1}+\Delta}{P \Gamma(\alpha) \Gamma(\alpha-\beta) \Gamma(\alpha+\beta)}, & 0 \leq s \leq t \leq 1, s \leq \eta, \\ \frac{\Delta}{P \Gamma(\alpha) \Gamma(\alpha-\beta) \Gamma(\alpha+\beta)}, & 0 \leq t \leq s \leq \eta \leq 1 \\ \frac{-P \Gamma(\alpha-\beta) \Gamma(\alpha+\beta)(t-s)^{\alpha-1}+\Lambda}{P \Gamma(\alpha) \Gamma(\alpha-\beta) \Gamma(\alpha+\beta)}, & 0 \leq \eta \leq s \leq t \leq 1 \\ \frac{\Gamma(\alpha) \Gamma(\alpha+\beta)(1-s)^{\alpha-\beta-1} t^{\alpha-1}}{P \Gamma(\alpha) \Gamma(\alpha-\beta) \Gamma(\alpha+\beta)}, & 0 \leq t \leq s \leq 1, s \geq \eta,\end{cases}
$$

where

$$
\Delta=t^{\alpha-1}\left[\Gamma(\alpha) \Gamma(\alpha+\beta)(1-s)^{\alpha-\beta-1}-\lambda \Gamma(\alpha) \Gamma(\alpha-\beta)(\eta-s)^{\alpha+\beta-1}\right],
$$

and

$$
\Lambda=\Gamma(\alpha+\beta) \Gamma(\alpha)(1-s)^{\alpha-\beta-1} t^{\alpha-1},
$$

where

$$
P=\frac{\Gamma(\alpha)}{\Gamma(\alpha-\beta)}-\frac{\lambda \Gamma(\alpha)}{\Gamma(\alpha+\beta)} \eta^{\alpha+\beta-1}
$$

Proof. In view of Lemma 2.5, the general solution for the above equation in (2.1) is

$$
u(t)=-\frac{1}{\Gamma(\alpha)} \int_{0}^{t}(t-s)^{\alpha-1} y(s) d s+c_{1} t^{\alpha-1}+c_{2} t^{\alpha-2}+c_{3} t^{\alpha-3}+C_{4} t^{\alpha-4},
$$

where $c_{1}, c_{2}, c_{3}, c_{4} \in \mathbb{R}$.

The boundary condition $u(0)=u^{\prime}(0)=u^{\prime \prime}(0)=0$, implies that $c_{2}=c_{3}=c_{4}=0$. Thus

$$
u(t)=-\frac{1}{\Gamma(\alpha)} \int_{0}^{t}(t-s)^{\alpha-1} y(s) d s+c_{1} t^{\alpha-1} .
$$


By (2.4) and Lemma 2.4, we get

$$
D_{0^{+}}^{\beta} u(t)=\frac{1}{\Gamma(\alpha-\beta)}\left[c_{1} \Gamma(\alpha) t^{\alpha-\beta-1}-\int_{0}^{t}(t-s)^{\alpha-\beta-1} y(s) d s\right] .
$$

In view of boundary condition $D_{0^{+}}^{\beta} u(1)=\lambda I_{0^{+}}^{\beta} u(\eta)$, we conclude that

$$
c_{1}=\frac{1}{P}\left[\frac{1}{\Gamma(\alpha-\beta)} \int_{0}^{1}(1-s)^{\alpha-\beta-1} y(s) d s-\frac{\lambda}{\Gamma(\alpha+\beta)} \int_{0}^{\eta}(\eta-s)^{\alpha+\beta-1} y(s) d s\right] .
$$

Therefore, the unique solution of the problem (2.1) is given by

$$
\begin{gathered}
u(t)=\frac{t^{\alpha-1}}{P \Gamma(\alpha-\beta)} \int_{0}^{1}(1-s)^{\alpha-\beta-1} y(s) d s-\frac{\lambda t^{\alpha-1}}{P \Gamma(\alpha+\beta)} \int_{0}^{\eta}(\eta-s)^{\alpha+\beta-1} y(s) d s \\
-\frac{1}{\Gamma(\alpha)} \int_{0}^{t}(t-s)^{\alpha-1} y(s) d s .
\end{gathered}
$$

For $t \leq \eta$, one has

$$
\begin{aligned}
& u(t)=\frac{t^{\alpha-1}}{P \Gamma(\alpha-\beta)}\left[\int_{0}^{t}(1-s)^{\alpha-\beta-1} y(s) d s+\int_{t}^{\eta}(1-s)^{\alpha-\beta-1} y(s) d s\right. \\
& \left.+\int_{\eta}^{1}(1-s)^{\alpha-\beta-1} y(s) d s\right]-\frac{1}{\Gamma(\alpha)} \int_{0}^{t}(t-s)^{\alpha-1} y(s) d s \\
& -\frac{\lambda t^{\alpha-1}}{P \Gamma(\alpha+\beta)}\left[\int_{0}^{t}(\eta-s)^{\alpha+\beta-1} y(s) d s+\int_{t}^{\eta}(\eta-s)^{\alpha+\beta-1} y(s) d s\right] \\
& =\int_{0}^{t} \frac{-P \Gamma(\alpha-\beta) \Gamma(\alpha+\beta)(t-s)^{\alpha-1}+\Delta}{P \Gamma(\alpha) \Gamma(\alpha+\beta) \Gamma(\alpha-\beta)} y(s) d s \\
& +\int_{t}^{\eta} \frac{\Delta}{P \Gamma(\alpha) \Gamma(\alpha+\beta) \Gamma(\alpha-\beta)} y(s) d s \\
& +\int_{\eta}^{1} \frac{\Gamma(\alpha) \Gamma(\alpha+\beta)(1-s)^{\alpha-\beta-1} t^{\alpha-1}}{P \Gamma(\alpha) \Gamma(\alpha+\beta) \Gamma(\alpha-\beta)} y(s) d s \\
& =\int_{0}^{1} G(t, s) y(s) d s .
\end{aligned}
$$


For $t \geq \eta$, one has

$$
\begin{gathered}
u(t)=\int_{0}^{\eta} \frac{-P \Gamma(\alpha-\beta) \Gamma(\alpha+\beta)(t-s)^{\alpha-1}+\Delta}{P \Gamma(\alpha) \Gamma(\alpha+\beta) \Gamma(\alpha-\beta)} y(s) d s \\
+\int_{\eta}^{t} \frac{-P \Gamma(\alpha-\beta) \Gamma(\alpha+\beta)(t-s)^{\alpha-1}+\Gamma(\alpha) \Gamma(\alpha+\beta)(1-s)^{\alpha-\beta-1} t^{\alpha-1}}{P \Gamma(\alpha) \Gamma(\alpha+\beta) \Gamma(\alpha-\beta)} y(s) d s \\
+\int_{t}^{1} \frac{\Gamma(\alpha) \Gamma(\alpha+\beta)(1-s)^{\alpha-\beta-1} t^{\alpha-1}}{P \Gamma(\alpha) \Gamma(\alpha+\beta) \Gamma(\alpha-\beta)} y(s) d s \\
=\int_{0}^{1} G(t, s) y(s) d s .
\end{gathered}
$$

The proof is complete.

We need some properties of function $G(t, s)$ to establish the existence of positive solutions.

Lemma 2.10. The Green's function $G(t, s)$ has the following properties:

(i) The function $G(t, s)$ is continuous on $[0,1] \times[0,1]$.

(ii) $G(t, s)>0$ for all $s \in(0,1)$,

(iii) for all $t, s \in(0,1)$, we have $G(t, s) \leq G(1, s)$,

$(i v)$ there exists a positive function $\gamma(s) \in C(0,1)$ such that

$$
\min _{\eta \leq t \leq 1} G(t, s) \geq \gamma(s) \max _{0 \leq t \leq 1} G(t, s)=\eta^{\alpha-1} G(1, s), 0<s<1 .
$$

Proof. It is easy to prove $(i)$. Now, we prove $(i i)-(i v)$. Let

$$
g_{1}(t, s)=\frac{\Delta-P \Gamma(\alpha-\beta) \Gamma(\alpha+\beta)(t-s)^{\alpha-1}}{P \Gamma(\alpha) \Gamma(\alpha+\beta) \Gamma(\alpha-\beta)},
$$

where $\Delta$ defined above.

$$
\begin{gathered}
g_{2}(t, s)=\frac{t^{\alpha-1} \Gamma(\alpha) \Gamma(\alpha+\beta)(1-s)^{\alpha-\beta-1}-P \Gamma(\alpha-\beta) \Gamma(\alpha+\beta)(t-s)^{\alpha-1}}{P \Gamma(\alpha) \Gamma(\alpha+\beta) \Gamma(\alpha-\beta)} \\
g_{3}(t, s)=\frac{t^{\alpha-1} \Gamma(\alpha)\left(\Gamma(\alpha+\beta)(1-s)^{\alpha-\beta-1}-\lambda \Gamma(\alpha-\beta)(\eta-s)^{\alpha+\beta-1}\right)}{P \Gamma(\alpha) \Gamma(\alpha+\beta) \Gamma(\alpha-\beta)} \\
g_{4}(t, s)=\frac{t^{\alpha-1} \Gamma(\alpha) \Gamma(\alpha+\beta)(1-s)^{\alpha-\beta-1}}{P \Gamma(\alpha) \Gamma(\alpha+\beta) \Gamma(\alpha-\beta)} .
\end{gathered}
$$

We will first show that

$$
g_{1}(t, s)>0,0 \leq \min \{t, \eta\}<1 .
$$

To simplify we introduce the abbreviation

$$
\triangle_{1}=t^{\alpha-1} \Gamma(\alpha) \Gamma(\alpha+\beta)(1-s)^{\alpha-\beta-1} .
$$


We can rewrite $\triangle_{1}$ as

$$
\begin{gathered}
\triangle_{1}=t^{\alpha-1} \Gamma(\alpha) \Gamma(\alpha+\beta)\left(\frac{\Gamma(\alpha-\beta)}{\Gamma(\alpha-\beta)}-\frac{\lambda \Gamma(\alpha-\beta)}{\Gamma(\alpha+\beta)} \eta^{\alpha+\beta-1}\right. \\
\left.+\frac{\lambda \Gamma(\alpha-\beta)}{\Gamma(\alpha+\beta)} \eta^{\alpha+\beta-1}\right)(1-s)^{\alpha-\beta-1} \\
=t^{\alpha-1} \Gamma(\alpha-\beta) \Gamma(\alpha+\beta)\left(\frac{\Gamma(\alpha)}{\Gamma(\alpha-\beta)}-\frac{\lambda \Gamma(\alpha)}{\Gamma(\alpha+\beta)} \eta^{\alpha+\beta-1}\right. \\
\left.\quad+\frac{\lambda \Gamma(\alpha)}{\Gamma(\alpha+\beta)} \eta^{\alpha+\beta-1}\right)(1-s)^{\alpha-\beta-1} \\
=t^{\alpha-1} \Gamma(\alpha-\beta) \Gamma(\alpha+\beta)\left(P+\frac{\lambda \Gamma(\alpha) \eta^{\alpha+\beta-1}}{\Gamma(\alpha+\beta)}\right)(1-s)^{\alpha-\beta-1} \\
\lambda t^{\alpha-1} \Gamma(\alpha) \Gamma(\alpha-\beta)(\eta-s)^{\alpha+\beta-1}=\lambda t^{\alpha-1} \Gamma(\alpha) \Gamma(\alpha-\beta) \eta^{\alpha+\beta-1}\left(1-\frac{s}{\eta}\right)^{\alpha+\beta-1}
\end{gathered}
$$

and

$$
P \Gamma(\alpha-\beta) \Gamma(\alpha+\beta)(t-s)^{\alpha-1}=P t^{\alpha-1} \Gamma(\alpha-\beta) \Gamma(\alpha+\beta)\left(1-\frac{s}{t}\right)^{\alpha-1} .
$$

Thus

$$
\begin{gathered}
g_{1}(t, s)=Q\left\{P t^{\alpha-1} \Gamma(\alpha-\beta) \Gamma(\alpha+\beta)\left[(1-s)^{\alpha-\beta-1}-\left(1-\frac{s}{t}\right)^{\alpha-1}\right]\right. \\
\left.+\lambda t^{\alpha-1} \Gamma(\alpha) \Gamma(\alpha-\beta)\left[\eta^{\alpha-\beta-1}(1-s)^{\alpha-\beta-1}-\eta^{\alpha+\beta-1}\left(1-\frac{s}{\eta}\right)^{\alpha+\beta-1}\right]\right\} \\
>Q\left\{P t^{\alpha-1} \Gamma(\alpha-\beta) \Gamma(\alpha+\beta)\left[(1-s)^{\alpha-1}-\left(1-\frac{s}{t}\right)^{\alpha-1}\right]\right. \\
\left.+\lambda t^{\alpha-1} \Gamma(\alpha) \Gamma(\alpha-\beta)\left[\eta^{\alpha+\beta-1}(1-s)^{\alpha+\beta-1}-\eta^{\alpha+\beta-1}\left(1-\frac{s}{\eta}\right)^{\alpha+\beta-1}\right]\right\} \\
>Q\left\{P t^{\alpha-1} \Gamma(\alpha-\beta) \Gamma(\alpha+\beta)\left[(1-s)^{\alpha-1}-(1-s)^{\alpha-1}\right]\right. \\
\left.+\lambda t^{\alpha-1} \Gamma(\alpha) \Gamma(\alpha-\beta) \eta^{\alpha+\beta-1}\left[(1-s)^{\alpha+\beta-1}-(1-s)^{\alpha+\beta-1}\right]\right\}=0,
\end{gathered}
$$

where $Q=\frac{1}{P \Gamma(\alpha) \Gamma(\alpha-\beta) \Gamma(\alpha+\beta)}$.

We deduce that $g_{1}(t, s)>0,0 \leq \min \{t, \eta\}<1$.

By the similar argument we can conclude that

$$
g_{2}(t, s)>0,0<\eta \leq s \leq t \leq 1, \quad g_{3}(t, s)>0,0 \leq t \leq s \leq \eta \leq 1,
$$

and

$$
g_{4}(t, s)>0,0 \leq \max \{s, \eta\} \leq s \leq 1 .
$$

Therefore $G(t, s)>0$ for any $t, s \in(0,1)$. 
Now, we show that $G(t, s) \leq G(1, s)$ for any $t, s \in(0,1)$.

Let $h_{1}(t, s)=g_{1}(t, s) \Gamma(\alpha) \Gamma(\alpha-\beta) \Gamma(\alpha+\beta)$. Then, as the above argument but for the derivative of $h_{1}(t, s)$ with respect to $t$ on $[s, 1]$, we have

$$
\begin{gathered}
\frac{\partial h_{1}(t, s)}{\partial t}=\frac{(\alpha-1) t^{\alpha-2}}{P}\left\{P \Gamma(\alpha-\beta) \Gamma(\alpha+\beta)\left[(1-s)^{\alpha-\beta-1}-\left(1-\frac{s}{t}\right)^{\alpha-2}\right]\right. \\
\left.+\lambda \Gamma(\alpha) \Gamma(\alpha-\beta)\left[\eta^{\alpha-\beta-1}(1-s)^{\alpha-\beta-1}-\eta^{\alpha+\beta-1}\left(1-\frac{s}{\eta}\right)^{\alpha+\beta-1}\right]\right\} \\
>\frac{(\alpha-1) t^{\alpha-2}}{P}\left\{P \Gamma(\alpha-\beta) \Gamma(\alpha+\beta)\left[(1-s)^{\alpha-2}-(1-s)^{\alpha-2}\right]\right. \\
\left.+\lambda t^{\alpha-1} \Gamma(\alpha) \Gamma(\alpha-\beta) \eta^{\alpha+\beta-1}\left[(1-s)^{\alpha+\beta-1}-(1-s)^{\alpha+\beta-1}\right]\right\}=0,
\end{gathered}
$$

so, we have $\frac{h_{1}(t, s)}{\partial t}>0$, then $g_{1}(t, s)$ is increasing with respect to $t$ on $[s, 1]$.

Next, we show that $g_{2}(t, s)$ is increasing with respect to $t$ on $[s, 1]$.

Let $h_{2}(t, s)=g_{2}(t, s) \Gamma(\alpha) \Gamma(\alpha-\beta) \Gamma(\alpha+\beta)$. Then, we have

$$
\begin{gathered}
\frac{\partial h_{2}(t, s)}{\partial t}=\frac{(\alpha-1) t^{\alpha-2}}{P}\left\{\Gamma(\alpha+\beta)\left[\Gamma(\alpha)(1-s)^{\alpha-\beta-1}-P \Gamma(\alpha-\beta)\left(1-\frac{s}{t}\right)^{\alpha-2}\right]\right\} \\
\geq \frac{(\alpha-1) t^{\alpha-2}}{P}\left\{\Gamma(\alpha+\beta)\left[\Gamma(\alpha)(1-s)^{\alpha-\beta-1}-P \Gamma(\alpha-\beta)(1-s)^{\alpha-2}\right]\right\} \\
\geq \frac{(\alpha-1)(1-s)^{\alpha-2} t^{\alpha-2}}{P}\left\{\Gamma(\alpha+\beta)\left[\Gamma(\alpha)(1-s)^{1-\beta}-P \Gamma(\alpha-\beta)\right]\right\} \\
=\frac{(\alpha-1)(t(1-s))^{\alpha-2}}{P}\left\{\Gamma(\alpha+\beta)\left[\Gamma(\alpha)(1-s)^{1-\beta}-P \Gamma(\alpha-\beta)\right]\right\} \\
=\frac{(\alpha-1)(t(1-s))^{\alpha-2}}{P}\left\{\Gamma(\alpha+\beta) \Gamma(\alpha)(1-s)^{1-\beta}+\lambda \Gamma(\alpha) \Gamma(\alpha-\beta) \eta^{\alpha+\beta-1}\right. \\
\quad-\Gamma(\alpha) \Gamma(\alpha+\beta)\} \\
\geq \frac{(\alpha-1)(t(1-s))^{\alpha-2}}{P}\left\{\Gamma(\alpha) \Gamma(\alpha+\beta)\left[(1-s)^{1-\beta}-1\right]\right\} \geq 0,
\end{gathered}
$$

so, we have $\frac{h_{2}(t, s)}{\partial t}>0$, then $g_{2}(t, s)$ is increasing with respect to $t$ on $[s, 1]$.

Then, we conclude that $G(t, s)$ is increasing with respect to $t$ on $[s, 1]$. Hence, $G(t, s) \leq G(1, s)$ for $s, t \in[0,1]$.

On the hand, we know that

$$
\begin{aligned}
\min _{\eta \leq t \leq 1} G(t, s)= & \left\{\begin{array}{l}
\min _{\eta \leq t \leq 1}\left\{g_{1}(t, s), g_{3}(t, s)\right\}, 0 \leq s \leq \eta \\
\min _{\eta \leq t \leq 1}\left\{g_{2}(t, s), g_{4}(t, s)\right\}, \eta \leq s \leq 1
\end{array}\right. \\
& =\left\{\begin{array}{l}
g_{1}(\eta, s), 0 \leq s \leq \eta \\
g_{2}(\eta, s), \eta \leq s \leq 1 .
\end{array}\right.
\end{aligned}
$$

Let

$$
\gamma(s) \leq\left\{\begin{array}{l}
\frac{g_{1}(\eta, s)}{G(1, s)}, 0<s \leq \eta, \\
\frac{g_{2}(\eta, s)}{G(1, s)}, \eta<s \leq 1,
\end{array}\right.
$$


where

$$
\begin{gathered}
G(1, s)=\left\{\begin{array}{l}
g_{1}(1, s), 0 \leq s \leq \eta, \\
g_{2}(1, s), \eta \leq s \leq 1 .
\end{array}\right. \\
= \begin{cases}\frac{\Gamma(\alpha)\left(\Gamma(\alpha+\beta)(1-s)^{\alpha-\beta-1}-\lambda \Gamma(\alpha-\beta)(\eta-s)^{\alpha+\beta-1}\right)-P \Gamma(\alpha-\beta) \Gamma(\alpha+\beta)(1-s)^{\alpha-1}}{P \Gamma(\alpha) \Gamma(\alpha+\beta) \Gamma(\alpha-\beta)}, & 0 \leq s \leq \eta \\
\frac{\Gamma(\alpha) \Gamma(\alpha+\beta)(1-s)^{\alpha-\beta-1}-P \Gamma(\alpha-\beta) \Gamma(\alpha+\beta)(1-s)^{\alpha-1}}{P \Gamma(\alpha) \Gamma(\alpha-\beta) \Gamma(\alpha+\beta)}, & \eta \leq s \leq 1 .\end{cases}
\end{gathered}
$$

Therefore, we have

$$
\gamma(s)=\eta^{\alpha-1} \in(0,1)
$$

Then

$$
\min _{\eta \leq t \leq 1} G(t, s) \geq \gamma(s) \max _{0 \leq t \leq 1} G(t, s)=\eta^{\alpha-1} G(1, s), 0<s<1 .
$$

The proof is complete.

\section{Existence results}

We shall consider the Banach space $E=C[0,1]$ equipped with the norm

$$
\|u\|=\max _{0 \leq t \leq 1}|u(t)|
$$

and let a closed cone $K \subset E$ by

$$
K=\{u \in E: u(t) \geq 0, t \in[0,1]\},
$$

where 0 is the the zero function. Obviously, $K$ is a reproducing cone of $E$.

Define the operator $T: K \rightarrow K$ and the linear operator $L: K \rightarrow K$ as follows

$$
T(u)(t)=\int_{0}^{1} G(t, s) a(s) f(s, u(s)) d s, \quad t \in[0,1],
$$

and

$$
L(u)(t)=\int_{0}^{1} G(t, s) a(s) u(s) d s, \quad t \in[0,1],
$$

where $G(t, s)$ is given by $(2.3)$. It is not hard to see that fixed points of operator $T$ coincide with the solutions to the problem $(1.1)-(1.2)$.

First, for the existence results of problem (1.1) - (1.2), we need the following assumptions.

$\left(H_{1}\right) f:[0,1] \times[0, \infty) \rightarrow[0, \infty)$ is continuous function, $\left(H_{2}\right) a(\cdot) \in L^{1}(0,1)$ is a nonnegative function, $a(t)$ does not vanish identically on any subinterval of $[0,1]$ and $0<\int_{0}^{1} a(s)(1-s)^{\alpha-\beta-1} s^{\alpha-1} d s<\infty$.

Lemma 3.1. Assume $\left(H_{1}\right)$ and $\left(H_{2}\right)$ hold. Then the operators $T: K \rightarrow K$ and $L: K \rightarrow K$ are completely continuous. 
Proof. For any $u \in K$, it follows from $\left(H_{1}\right),\left(H_{2}\right)$ and Lemma 2.10, $T(u)(t) \geq 0, t \in$ $[0,1]$. So, $T: K \rightarrow K$ and $L: K \rightarrow K$ are continuous.

Let $\Phi \subset K$ be bounded .i.e., there exists a positive constant $M$ such that $f(t, u) \leq M$ for all $t \in[0,1], u \in \Phi$. Then, It follows from (3.1) that

$$
\begin{aligned}
& |T u(t)| \leq \frac{M t^{\alpha-1}}{P \Gamma(\alpha-\beta)} \int_{0}^{1}(1-s)^{\alpha-\beta-1} a(s) d s+\frac{M}{\Gamma(\alpha)} \int_{0}^{t}(t-s)^{\alpha-1} a(s) d s \\
& +\frac{\lambda M t^{\alpha-1}}{P \Gamma(\alpha+\beta)} \int_{0}^{\eta}(\eta-s)^{\alpha+\beta-1} a(s) d s \\
& \leq \frac{M}{P \Gamma(\alpha-\beta)} \int_{0}^{1} a(s) d s+\frac{M}{\Gamma(\alpha)} \int_{0}^{1} a(s) d s \\
& +\frac{\lambda M}{P \Gamma(\alpha+\beta)} \int_{0}^{1} a(s) d s \\
& \leq \frac{M(\Gamma(\alpha) \Gamma(\alpha+\beta)+P \Gamma(\alpha+\beta) \Gamma(\alpha-\beta)+\lambda \Gamma(\alpha) \Gamma(\alpha-\beta))}{P \Gamma(\alpha) \Gamma(\alpha+\beta) \Gamma(\alpha-\beta)} \int_{0}^{1} a(s) d s .
\end{aligned}
$$

Thus $\|T u\|<\infty$ for all $u \in \Phi$. Hence, $\{T u, u \in \Phi\}$ is bounded.

Now, we show that $T$ maps bounded sets into equicontinuous sets of $K$.

Let $t_{1}, t_{2} \in[0,1]$ with $t_{1}<t_{2}$ and $u \in \Phi$ is a bounded set of $K$. Then

$$
\begin{gathered}
\left|T u\left(t_{2}\right)-T u\left(t_{1}\right)\right| \leq \mid \frac{t_{2}^{\alpha-1}}{P \Gamma(\alpha-\beta)} \int_{0}^{1}(1-s)^{\alpha-\beta-1} a(s) f(s, u(s)) d s \\
-\frac{t_{1}^{\alpha-1}}{P \Gamma(\alpha-\beta)} \int_{0}^{1}(1-s)^{\alpha-\beta-1} a(s) f(s, u(s)) d s \mid \\
+\left|\frac{t_{1}^{\alpha-1}}{\Gamma(\alpha)} \int_{0}^{t_{1}}\left(t_{1}-s\right)^{\alpha-1} a(s) f(s, u(s)) d s-\frac{t_{2}^{\alpha-1}}{\Gamma(\alpha)} \int_{0}^{t_{2}}\left(t_{2}-s\right)^{\alpha-1} a(s) f(s, u(s)) d s\right| \\
+\mid \frac{\lambda t_{1}^{\alpha-1}}{P \Gamma(\alpha+\beta)} \int_{0}^{\eta}(\eta-s)^{\alpha-1} a(s) f(s, u(s)) d s \\
\quad-\frac{\lambda t_{2}^{\alpha-1}}{P \Gamma(\alpha+\beta)} \int_{0}^{\eta}(\eta-s)^{\alpha-1} a(s) f(s, u(s)) d s \mid \\
\leq \frac{M\left(t_{2}^{\alpha-1}-t_{1}^{\alpha-1}\right)}{P \Gamma(\alpha-\beta)} \int_{0}^{1} a(s) d s+\frac{\lambda M\left(t_{2}^{\alpha-1}-t_{1}^{\alpha-1}\right)}{P \Gamma(\alpha-\beta)} \int_{0}^{1} a(s) d s
\end{gathered}
$$




$$
+\frac{M\left(t_{1}^{\alpha-1}-t_{2}^{\alpha-1}\right)}{\Gamma(\alpha)}\left|\int_{0}^{t_{1}}\left(t_{1}-s\right)^{\alpha-1} a(s) d s\right|+\frac{M t_{2}^{\alpha-1}}{\Gamma(\alpha)}\left|\int_{t_{1}}^{t_{2}}\left(t_{2}-s\right)^{\alpha-1} a(s) d s\right| .
$$

Obviously, the right hand side of the above inequality tends to zero as $t_{2} \rightarrow t_{1}$. Thus $\left\|(T u)\left(t_{2}\right)-(T u)\left(t_{1}\right)\right\| \rightarrow 0$, as $t_{2} \rightarrow t_{1}$. This shows that the operator $T$ is completely continuous, by the Arzela-Ascoli theorem.

By the same method we can get that $L: K \rightarrow K$ is a completely continuous operator. The proof is complete.

Now, we present the existence result for the boundary value problem $(1.1)-(1.2)$ via Banach contraction principle.

Theorem 3.2. Assume $\left(H_{1}\right)$ and $\left(H_{2}\right)$ hold. Suppose that $f:[0,1] \times[0, \infty) \rightarrow[0, \infty)$ be a continuous function satisfying the condition

$\left(H_{3}\right)|f(t, u)-f(t, v)| \leq l|u-v|$, for $t \in[0,1], l>0$ and $u, v \in[0,+\infty)$.

If $0<\int_{0}^{1} G(1, s) a(s) d s<1$, then the boundary value problem (1.1) - (1.2) has a unique positive solution on $[0,1]$.

Proof. As the first step, by Lemma 2.9 we know that $T: K \rightarrow K$.

Now, let $u, v \in K$ and for each $t \in[0,1]$, it follows from assumption $\left(H_{3}\right)$ that

$$
\begin{gathered}
\|T u(t)-T v(t)\|=\max _{t \in[0,1]}|T u(t)-T v(t)| \\
\leq \int_{0}^{1} G(t, s) a(s)|f(s, u(s))-f(s, v(s))| d s \\
\leq l \int_{0}^{1} G(1, s) a(s)|u(s)-v(s)| d s \\
\leq l \int_{0}^{1} G(1, s) a(s) d s\|u-v\| .
\end{gathered}
$$

Thus,

$$
\|(T u)-(T v)\| \leq l \int_{0}^{1} G(1, s) a(s) d s\|u-v\| .
$$

Since $l \int_{0}^{1} G(1, s) a(s) d s<1$, so $T$ s a contraction. Hence it follows by Banach's contraction principle that the boundary value problem (1.1) - (1.2) has a unique positive solution on $[0,1]$. The proof is complete.

Now, we are in a position to study the existence of solutions for the boundary value problem $(1.1)-(1.2)$ by applying the fixed point index theory.

Lemma 3.3. Assume $\left(H_{1}\right)$ and $\left(H_{2}\right)$ hold. Then the spectral radius of the operator $L$ is positive that is $r(L)>0$. 
Proof. Take $u(t)=t^{\alpha-1} \in E$. Then $\|u\|=1$. We have

$$
\begin{aligned}
& L u(t)=\frac{t^{\alpha-1}}{P \Gamma(\alpha-\beta)} \int_{0}^{1}(1-s)^{\alpha-\beta-1} a(s) u(s) d s \\
& -\frac{\lambda t^{\alpha-1}}{P \Gamma(\alpha+\beta)} \int_{0}^{\eta}(\eta-s)^{\alpha+\beta-1} a(s) u(s) d s-\frac{1}{\Gamma(\alpha)} \int_{0}^{t}(t-s)^{\alpha-1} a(s) u(s) d s \\
& =\frac{t^{\alpha-1}}{P \Gamma(\alpha-\beta)} \int_{0}^{1}(1-s)^{\alpha-\beta-1} a(s) s^{\alpha-1} d s \\
& -\frac{\lambda t^{\alpha-1} \eta^{\alpha+\beta-1}}{P \Gamma(\alpha+\beta)} \int_{0}^{\eta}\left(1-\frac{s}{\eta}\right)^{\alpha+\beta-1} a(s) s^{\alpha-1} d s-\frac{t^{\alpha-1}}{\Gamma(\alpha)} \int_{0}^{t}\left(1-\frac{s}{t}\right)^{\alpha-1} a(s) s^{\alpha-1} d s \\
& =t^{\alpha-1}\left\{-\frac{\lambda \eta^{\alpha+\beta-1}}{P \Gamma(\alpha+\beta)} \int_{0}^{\eta}\left(1-\frac{s}{\eta}\right)^{\alpha+\beta-1} a(s) s^{\alpha-1} d s\right. \\
& \left.+\frac{1}{P \Gamma(\alpha-\beta)} \int_{0}^{1}(1-s)^{\alpha-\beta-1} a(s) s^{\alpha-1} d s-\frac{1}{\Gamma(\alpha)} \int_{0}^{t}\left(1-\frac{s}{t}\right)^{\alpha-1} a(s) s^{\alpha-1} d s\right\} \\
& >t^{\alpha-1}\left\{-\frac{\lambda \eta^{\alpha+\beta-1}}{P \Gamma(\alpha+\beta)} \int_{0}^{1}\left(1-\frac{s}{\eta}\right)^{\alpha+\beta-1} a(s) s^{\alpha-1} d s\right. \\
& \left.+\frac{1}{P \Gamma(\alpha-\beta)} \int_{0}^{1}(1-s)^{\alpha-\beta-1} a(s) s^{\alpha-1}-\frac{1}{\Gamma(\alpha)} \int_{0}^{1}\left(1-\frac{s}{t}\right)^{\alpha-1} a(s) s^{\alpha-1} d s\right\} \\
& >t^{\alpha-1}\left\{-\frac{\lambda \eta^{\alpha+\beta-1}}{P \Gamma(\alpha+\beta)} \int_{0}^{1}(1-s)^{\alpha+\beta-1} a(s) s^{\alpha-1} d s\right. \\
& \left.+\frac{1}{P \Gamma(\alpha-\beta)} \int_{0}^{1}(1-s)^{\alpha-\beta-1} a(s) s^{\alpha-1} d s-\frac{1}{\Gamma(\alpha)} \int_{0}^{1}(1-s)^{\alpha-1} a(s) s^{\alpha-1} d s\right\} \\
& =t^{\alpha-1}\left\{-\frac{1}{\Gamma(\alpha)} \int_{0}^{1}(1-s)^{\alpha-1} a(s) s^{\alpha-1} d s\right. \\
& \left.+\frac{1}{\Gamma(\alpha)} \int_{0}^{1}(1-s)^{\alpha-\beta-1} a(s) s^{\alpha-1} d s\right\}=\nu t^{\alpha-1}>0
\end{aligned}
$$


where

$$
\nu=-\frac{1}{\Gamma(\alpha)} \int_{0}^{1}(1-s)^{\alpha-1} a(s) s^{\alpha-1} d s+\frac{1}{\Gamma(\alpha)} \int_{0}^{1}(1-s)^{\alpha-\beta-1} a(s) s^{\alpha-1} d s .
$$

Since $L: K \rightarrow K$, according the monotonicity of $L$ and $\left(H_{2}\right)$, we deduce

$$
L^{2} u(t)=L(L u(t))>L\left(\nu t^{\alpha-1}\right)>\nu L\left(t^{\alpha-1}\right)>\nu^{2} t^{\alpha-1} .
$$

Repeating the process gives $L^{n} u(t)>\nu^{n} t^{\alpha-1}$. So, we get $\left\|L^{n}\right\|>\nu^{n}$. Hence

$$
\left\|L^{n}\right\|^{\frac{1}{n}}>\nu, \quad r(L)=\lim _{n \rightarrow \infty}\left\|L^{n}\right\|^{\frac{1}{n}}>\nu>0 .
$$

The proof is complete.

For convenience, we introduce the following notation:

$$
\begin{gathered}
f^{\infty}=\lim _{u \rightarrow \infty} \sup \max _{t \in[0,1]} \frac{f(t, u)}{u}, \\
f_{0}=\lim _{u \rightarrow 0^{+}} \inf \min _{t \in[0,1]} \frac{f(t, u)}{u}, \\
K_{c}=\{u \in K:\|u\|<c\}, \\
r(L)=\frac{1}{\mu}, \mu \in \mathbb{R}^{+} .
\end{gathered}
$$

Lemma 3.4. Assume $\left(H_{1}\right),\left(H_{2}\right)$ hold and $\mu<f_{0} \leq \infty$. Then there exists $\rho_{0}>0$ such that for $\rho \in\left(0, \rho_{0}\right]$, if $u \neq T u, u \in \partial K_{\rho}$, then $i\left(T, K_{\rho}, K\right)=0$.

Proof. It follows from $\mu<f_{0}$ that there exist $\varepsilon>0$ and $\rho_{0}>0$ such that for $t \in[0,1]$ and $0 \leq u \leq \rho_{0}$ we have

$$
f(t, u) \geq(\mu+\varepsilon) u .
$$

For $0<\rho<\rho_{0}$ assume that $u \neq T u, u \in \partial K_{\rho}$. By Lemma 2.7 and Lemma $2.8(i)$, we need only to prove that

$$
u \neq T u+\lambda \varphi, \lambda>0,
$$

where $\varphi \in K \backslash\{0\}$ with $L \varphi=r(L) \varphi$.

Otherwise, there exist $u_{0} \in \partial K_{\rho}$ and $\lambda_{0}>0$ such that

$$
u_{0} \neq T u_{0}+\lambda_{0} \varphi \text {. }
$$

Then $u_{0} \geq T u_{0}$ and $u_{0} \geq \lambda_{0} \varphi$.

From (2.1), we get

$$
T u_{0}(t)=\int_{0}^{1} G(t, s) a(s) f\left(s, u_{0}(s)\right) d s \geq(\mu+\varepsilon) L u_{0}(t) .
$$

Considering $u_{0} \geq \lambda_{0} \varphi$, we have

$$
l u_{0} \geq \lambda_{0} L \varphi .
$$

For $L \varphi=r(L) \varphi,(\mu+\varepsilon) r(L)>1$, so that $(\mu+\varepsilon) r(L) \varphi>\varphi$.

Thus, we can conclude $T u_{0} \geq(\mu+\varepsilon) \lambda_{0} L \varphi>\lambda_{0} \varphi$. 
Together with the boundary conditions in (2.1), we have $u_{0} \geq 2 \lambda_{0} \varphi$. By (3.3), we obtain $T u_{0} \geq 2 \lambda_{0} \varphi$. Thus, $u_{0} \geq 3 \lambda_{0} \varphi$.

Repeating this process, we get that $u_{0} \geq n \lambda_{0} \varphi$. Hence, we have $\left\|u_{0}\right\| \geq n \lambda_{0}\|\varphi\| \rightarrow \infty$ as $n \rightarrow \infty$. This is a contradiction.

It follows from Lemma $2.8(i i)$ that $i\left(T, K_{\rho}, K\right)=0$ for $\rho \in\left(0, \rho_{0}\right]$. The proof is complete.

Lemma 3.5. Assume $\left(H_{1}\right),\left(H_{2}\right)$ hold and $0 \leq f^{\infty}<\mu$. Then there exists $\tau_{0}>0$ such that for $\tau>\tau_{0}$, if $\lambda u \neq T u, u \in \partial K_{\tau}$, then $i\left(T, K_{\rho}, K\right)=1$.

Proof. let $\varepsilon>0$ satisfy $f^{\infty}<\mu-\varepsilon$. Then there exist $\tau_{1}>0$ and such that for $u>\tau_{1}$ and $t \in[0,1]$, we have

$$
f(t, u) \leq(\mu-\varepsilon) u .
$$

Set $\Psi(t)=\max _{u \in\left[0, \tau_{1}\right]} f(t, u)$. Then, for all $u \in \mathbb{R}^{+}$and $t \in[0,1]$, we have

$$
f(t, u) \leq(\mu-\varepsilon) u+\Psi(t)
$$

Let

$$
F=\left\|\int_{0}^{1} G(t, s) a(s) \Psi(s) d s\right\|, \tau_{0}=\left\|\frac{F}{\mu-\varepsilon}\left(\frac{I}{\mu-\varepsilon}-L\right)^{-1}\right\| .
$$

Take $\tau>\tau_{0}$. We will show that $\lambda u \neq T u$, for all $u \in \partial K_{\tau}$ and $\lambda \geq 1$.

Otherwise, there exist $u_{0} \in \partial K_{\tau}$ and $\lambda_{0} \geq 1$ such that

$$
T u_{0}=\lambda_{0} u_{0} .
$$

Together with (3.7), we have

$$
u_{0} \leq \lambda u_{0}=T u_{0} \leq(\mu-\varepsilon) L u_{0}+F .
$$

Then $\frac{F}{\mu-\varepsilon} \geq\left(\frac{I}{\mu-\varepsilon}-L\right) u_{0}(t)$ for $t \in[0,1]$. So, $\frac{F}{\mu-\varepsilon}-\left(\frac{I}{\mu-\varepsilon}-L\right) u_{0}(t) \in K$.

It follows from $L(K) \subset K$ that $u_{0}(t) \leq \frac{F}{\mu-\varepsilon}\left(\frac{I}{\mu-\varepsilon}-L\right)^{-1} t \in[0,1]$. Therefore, we have $\left\|u_{0}\right\| \leq \tau_{0}<\tau$. This is a contradiction. Thus, we conclude that for all $u \in \partial K_{\tau}$ and $\lambda \geq 1$

$$
T u \neq \lambda u \text {. }
$$

It follows from Lemma $2.8(i i)$ that $i\left(T, K_{\tau}, K\right)=1$ for $\tau_{0}<\tau$.

The proof is complete.

Theorem 3.6. Assume $\left(H_{1}\right),\left(H_{2}\right)$ hold, $\mu<f_{0} \leq \infty$ and $0 \leq f^{\infty} \leq \mu$. Then, the boundary value problem $(1.1)-(1.2)$ has at least one positive solution on $[0,1]$.

Proof. It follows from $\mu<f_{0} \leq \infty$ and Lemma 3.4 that there exist $0<\rho<\tau$ such that either there exists $u \in \partial K_{\rho}$ with $u=T u$ or $i\left(T, K_{\rho}, K\right)=0$. From $0 \leq f^{\infty} \leq \mu$ and Lemma 3.5 there exists $\tau>0$ such that $i\left(T, K_{\tau}, K\right)=1$. Thus, we can conclude that $T$ has fixed point $u \in K$ with $\rho<\|u\|<\tau$ by the properties of index. Hence, the boundary value problem $(1.1)-(1.2)$ has at least one positive solution on $[0,1]$. The proof is complete. 
Now, we are in the position to present the third main results of this paper. The existence and the multiplicity result is based on the Leggett-Williams fixed point theorem.

Theorem 3.7. Assume $\left(H_{1}\right)$ and $\left(H_{2}\right)$ hold. Furthermore, suppose that there exist constants $0<d<l<c$ such that

$\left(H_{4}\right) \quad f(t, u)<M d,(t, u) \in[0,1] \times[0, d]$,

$\left(H_{5}\right) \quad f(t, u) \leq M c$, for $(t, u) \in[0,1] \times[0, c]$,

$\left(H_{6}\right) \quad f(t, u) \geq N l$, for $(t, u) \in[\eta, 1] \times[l, c]$,

where

$$
M=\left(\int_{0}^{1} a(s) G(1, s) d s\right)^{-1}
$$

and

$$
N=\left(\int_{\eta}^{1} a(s) \gamma(s) G(1, s) d s\right)^{-1}, \text { and } \gamma(s) \in(0,1)
$$

Then the boundary value problem (1.1) - (1.2) has at least three positive solutions $u_{1}, u_{2}$ and $u_{3}$ such that

$$
\left\|u_{1}\right\|<d, \quad l<\beta\left(u_{2}\right), \quad u_{3}>d \text { with } \beta\left(u_{3}\right)<l .
$$

Proof. Let $\beta(u)=\min _{t \in[\eta, 1]}|u(t)|$. Then $\beta(u)$ is nonnegative continuous concave functional on the cone $K$ satisfying $\beta(u) \leq\|u\|$ for all $u \in K$.

Let $u \in \bar{K}_{c}$, then $\|u\| \leq c$. It follows from $\left(H_{5}\right)$ and Lemma 2.10 (iii) that

$$
\begin{aligned}
|T u(t)| & =\left|\int_{0}^{1} G(t, s) a(s) f(s, u(s)) d s\right| \\
& \leq M c \int_{0}^{1} G(1, s) a(s) d s=c,
\end{aligned}
$$

which implies that $\|T u\| \leq c$, which shows that $T u \in \bar{K}_{c}$. Hence, we have shown that if $\left(H_{5}\right)$ holds, then $T$ maps $\bar{K}_{c}$ into $\bar{K}_{c}$ and by Lemma 3.1, $T$ is completely continuous.

If $u \in \bar{K}_{d}$, then it follows from $\left(H_{4}\right)$ and Lemma $2.10($ iii $)$ that

$$
\begin{aligned}
(T u)(t) & =\int_{0}^{1} G(t, s) a(s) f(s, u(s)) d s \\
& <M d \int_{0}^{1} G(1, s) a(s) d s=d .
\end{aligned}
$$


We verify that $\{u / K(\beta, l, r): \beta(u)>l\} \neq \phi$ and $\beta(T u)>l$ for all $u \in K(\beta, l, r)$. Take $\varphi_{0}(t)=\frac{l+r}{2}$, for $t \in[0,1]$. Then

$$
\varphi_{0} \in\{u / u \in K(\beta, l, r), \beta(u)>l\} .
$$

This shows that

$$
\{u / u \in K(\beta, l, r): \beta(u)>l\} \neq \phi .
$$

Finally, we assert that if $u \in K(\beta, l, c)$ and $\|T u\|>c$, then $\beta(T u)>l$.

Suppose $u \in K(\beta, l, c)$ and $\|u(t)\|>r, t \in[\eta, 1]$, then $\|u\|<c$. It follows from $\left(H_{6}\right)$ that

$$
\begin{aligned}
\beta(T u) & =\min _{t \in[\eta, 1]}(T u)(t) \\
& \geq \min _{t \in[\eta, 1]} \int_{0}^{1} G(t, s) a(s) f(s, u(s)) d s \\
& >N l \int_{\eta}^{1} G(1, s) a(s) \gamma(s) d s=l,
\end{aligned}
$$

which implies that $\beta(T u)>l$ for $u \in K(\beta, l, c)$.

To sum up, the hypotheses of Theorem 2.6 hold. Therefore, boundary value problem $(1.1)-(1.2)$ has at least three positive solutions $u_{1}, u_{2}$ and $u_{3}$ such that

$$
\left\|u_{1}\right\|<d, \quad l<\beta\left(u_{2}\right), \quad u_{3}>d \text { with } \beta\left(u_{3}\right)<l .
$$

The proof is complete.

We present two examples to illustrate the applicability of the results shown before.

Example 3.8. Consider the following boundary value problem

$$
\begin{gathered}
D_{0^{+}}^{\frac{7}{2}} u(t)+\frac{1}{(t+\cos t+3)^{2}}\left(\sin ^{2} t+\arctan (u)+\frac{|u|}{1+|u|}\right)=0, t \in(0,1), \\
u(0)=u^{\prime}(0)=u^{\prime \prime}(0)=0, D^{\frac{5}{2}} u(1)=\frac{1}{2} I_{0^{+}}^{\frac{5}{2}} u\left(\frac{1}{2}\right)
\end{gathered}
$$

where $\alpha=\frac{7}{2}, \beta=\frac{5}{2}, \lambda=\frac{1}{2}, \eta=\frac{1}{2}$ and

$$
f(t, u)=\frac{1}{(t+\cos t+3)^{2}}\left(\sin ^{2} t+\arctan (u)+\frac{|u|}{1+|u|}\right) .
$$

Clearly $l=\frac{2}{9}$ as $|f(t, u)-f(t, v)| \leq \frac{2}{9}|u-v|$.

We take $a(t)=1$. A simple calculation leads to $P \cong 1,32620$. 
Furthermore, by simple computation, we have

$$
\begin{aligned}
\frac{1}{M} & =\int_{0}^{1} a(s) G(1, s) d s \\
& =\frac{\Gamma(\alpha) \Gamma(\alpha+\beta) \int_{0}^{1} d s-P \Gamma(\alpha-\beta) \Gamma(\alpha+\beta) \int_{0}^{1}(1-s)^{\frac{5}{2}} d s}{P \Gamma(\alpha) \Gamma(\alpha-\beta) \Gamma(\alpha+\beta)} \\
& +\frac{\Gamma(\alpha) \Gamma(\alpha+\beta) \int_{\eta}^{1}(1-s) d s}{P \Gamma(\alpha) \Gamma(\alpha-\beta) \Gamma(\alpha+\beta)} \cong 0,27303
\end{aligned}
$$

so,

$$
0<l \int_{0}^{1} a(s) G(1, s) d s \leq \frac{2}{9}(0,27303) \cong 0,060673<1 .
$$

Thus all assumptions of Theorem 3.2 are satisfied. So, by the conclusion of Theorem 3.2 , problem $(3.9)-(3.10)$ has a unique solution on $[0,1]$.

Example 3.9. Consider the following boundary value problem

$$
\begin{array}{lr}
D_{0^{+}}^{\frac{7}{2}} u(t)+f(t, u(t))=0, & t \in(0,1), \\
u(0)=u^{\prime}(0)=u^{\prime \prime}(0)=0, D^{\frac{5}{2}} u(1)=\frac{1}{2} I_{0^{+}}^{\frac{5}{2}} u\left(\frac{1}{2}\right),
\end{array}
$$

where $\alpha=\frac{7}{2}, \beta=\frac{5}{2}, \lambda=\frac{1}{2}, \eta=\frac{1}{2}$, and here

$$
f(t, u)=\left\{\begin{array}{c}
10 u+t,(t, u) \in[0,1] \times[0,1] \\
10, \quad(t, u) \in[0,1] \times(1,+\infty) .
\end{array}\right.
$$

We take $a(t)=1$. We see that $f \in C([0,1] \times[0, \infty),[0, \infty))$, so, assumption $\left(H_{1}\right)$ satisfied. And

$$
0<\int_{0}^{1} a(s)(1-s)^{\alpha-\beta-1} s^{\alpha-1} d s=\int_{0}^{1}(1-s) s^{\frac{5}{2}} d s=\frac{4}{63}<\infty,
$$

so, assumption $\left(H_{2}\right)$ satisfied.

By simple calculation, we obtain $P \cong 1,32620, M \cong 3,66264$ and $N \cong 7218,14758$.

Choosing, $d=\frac{1}{4}, l=1$ and $c=3$, we have

$$
\begin{gathered}
f(t, u)=10 u+t \leq 3.5<M d \cong 14,65056,(t, u) \in[0,1] \times\left[0, \frac{1}{4}\right], \\
f(t, u)=10 \leq M l \cong 10,98792,(t, u) \in[0,1] \times(1,3]
\end{gathered}
$$

and

$$
f(t, u)=10 \geq N r \cong 9,00765,(t, u) \in\left[\frac{1}{2}, 1\right] \times(1,3] .
$$

Thus, all assumptions and conditions of Theorem 3.7 are satisfied. Hence Theorem 3.7 , implies that the problem $(3.11)-(3.12)$ has at least three solutions $u_{1}, u_{2}$ and $u_{3}$ such that

$$
\left\|u_{1}\right\|<d, \quad l<\beta\left(u_{2}\right), \quad u_{3}>d \text { with } \beta\left(u_{3}\right)<l .
$$




\section{References}

[1] Al-Refai, M., Hadjji, M., Monotone iterative sequences for nonlinear boundary value problems of fractional order, Nonlinear Anal., 74(2011), 3531-3539.

[2] Bai, Z.B., Sun, W.C., Zhang, W., Positive solutions for boundary value problems of singular fractional differential equations, Abstr. Appl. Anal. 2013, Art. ID 129640 (2013).

[3] Benaicha, S., Bouteraa, N., Existence of solutions for three-point boundary value problem for nonlinear fractional differential equations, Bull. Transil. Univ. Braşov Ser. III: Mathematics, Informatics, Physics, 10(59)(2017), no. 2, 31-48.

[4] Bouteraa, N., Benaicha, S., Existence of solutions for three-point boundary value problem for nonlinear fractional equations, An. Univ. Oradea, Fasc. Mat., 24(2017), no. 2, 109119.

[5] Bouteraa, N., Benaicha, S., Djourdem, H., Positive solutions for nonlinear fractional equation with nonlocal boundary conditions, Universal Journal of Mathematics and Applications, 1(2018), no. 1, 39-45.

[6] Bouteraa, N., Benaicha, S., The uniqueness of positive solution for higher-order nonlinear fractional differential equation with nonlocal boundary conditions, Advances in the Theory of Nonlinear and it Application, 2(2018), no. 2, 74-84.

[7] Bouteraa, N., Benaicha, S., The uniqueness of positive solution for nonlinear fractional differential equation with nonlocal boundary conditions, An. Univ. Oradea, Fasc. Mat., 24(2018), no. 2, 53-65.

[8] Cabada, A., Wang, G., Positive solutions of nonlinear fractional differential equations with integral boundary conditions, J. Math. Anal. Appl., 389(2012), no. 1, 403-411.

[9] Han, X.L., Gao, H.L., Existence of positive solutions for eigenvalue problem of nonlinear fractional differential equations, Adv. Differ. Equ., 66(2012).

[10] Kilbas, A.A., Srivastava, H.M., Trijullo, J.J., Theory and Applications of Fractional Differential Equations, Elsevier Science, Amsterdam, 2006.

[11] Lakshmikantham, V., Vatsala, A.S., General uniqueness and monotone iterative technique for fractional differential equations, Appl. Math. Lett., 21(8)(2008), 828-834.

[12] Li, C.F., Luo, X.N., Zhou, Y., Existence of positive solutions of the boundary value problem for nonlinear fractional differential equations, Comput. Math. Appl., 59(2010), 1363-1375.

[13] Liu, F., Burrage, K., Novel techniques in parameter estimation for fractional dynamycal models arising from biological systems, Comput. Math. Appl., 62(2011), no. 3, 822-833.

[14] Liu, S., Li, H., Dai, Q., Nonlinear fractional differential equations with nonlocal integral boundary conditions, Bound. Value. Prob., (2015), 11 pages.

[15] Miller, S., Ross, B., An Introduction to the Fractional Calculus and Fractional Differential Equations, John Wiley and Sons, Inc. New York, 1993.

[16] Samko, S.G., Kilbas, A.A., Marichev, O.I., Fractional Integrals and Derivatives: Theory and Applications, Gordon \& Breach, Yverdon, 1993.

[17] Sun, Y., Zhao, M., Positive solutions for a class of fractional differential equations with integral boundary conditions, Appl. Math. Lett., 34(2014), 17-21.

[18] Tan, J., Cheng, C., Fractional boundary value problems with Riemann-Liouville fractional derivatives, Bound. Value Probl., (2015), 14 pages.

[19] Xu, Y.F., Fractional boundary value problems with integral and anti-periodic boundary conditions, Bull. Malays. Math. Sci. Soc., 39(2016), 571-587. 
[20] Zeidler, E., Nonlinear Functional Analysis and its Applications. Fixed-Point Theorem, Springer, Berlin, 1985.

[21] Zhai, C., Hoo, M., Fixed point theorem for mixed monotone operators with perturbation and applications to fractional differential equation boundary value problems, Nonlinear Anal. TMA, 75(2012), 2542-2551.

[22] Zhang, X, Wang, L., Sun, Q., Existence of positive solutions for a class of nonlinear fractional differential equations with integral boundary conditions and a parameter, Appl. Math. Comput., 226(2014), 708-718.

Noureddine Bouteraa

Laboratory of Fundamental and Applied Mathematics of Oran (LMFAO),

University of Oran1, Ahmed Benbella,

Algeria

e-mail: bouteraa-27@hotmail.fr

Slimane Benaicha

Laboratory of Fundamental and Applied Mathematics of Oran (LMFAO),

University of Oran1, Ahmed Benbella,

Algeria

e-mail: slimanebenaicha@yahoo.fr 